IPMU 12-0233

ICRR-Report-642-2012-31

\title{
Extended analysis of CMB constraints on non-Gaussianity in isocurvature perturbations
}

\author{
Chiaki Hikage $^{a}$, Masahiro Kawasakib ${ }^{b, c}$, Toyokazu Sekiguchi ${ }^{d}$ and Tomo Takahashi ${ }^{e}$ \\ ${ }^{a}$ Kobayashi-Maskawa Institute, Nagoya University, Nagoya 464-8602, Japan \\ ${ }^{b}$ Institute for Cosmic Ray Research, University of Tokyo, Kashiwa 277-8582, Japan \\ ${ }^{c}$ Kavli Institute for the Physics and Mathematics of the Universe, University of Tokyo, \\ Kashiwa 277-8568, Japan \\ ${ }^{d}$ Department of Physics and Astrophysics, Nagoya University, Nagoya 464-8602, Japan \\ ${ }^{e}$ Department of Physics, Saga University, Saga 840-8502, Japan
}

\begin{abstract}
We study CMB constraints on non-Gaussianity from isocurvature perturbations of general types. Specifically, we study CDM/neutrino isocurvature perturbations which are uncorrelated or totally correlated with adiabatic ones. Using the data from the WMAP 7-year observation at $\mathrm{V}$ and $\mathrm{W}$ bands, we obtained optimal constraints on the nonlinearity parameters of adiabatic and isocurvature perturbations. Our result shows that primordial perturbations are consistent with Gaussian ones at around $2 \sigma$ level for above mentioned isocurvature modes.
\end{abstract}




\section{Introduction}

The adiabaticity of primordial density perturbations are one of the most important probes to understand the early Universe. Although current cosmological observations suggest that primordial fluctuations are almost adiabatic and the contribution from isocurvature fluctuations is severely constrained, once any deviation from the adiabatic fluctuations are detected even if it is tiny, it readily indicates that there are multiple sources of density fluctuations.

In the view of its importance, there have been many works on constraints on isocurvature fluctuations by using power spectrum [1-10]. However, current cosmological observations are so precise that its non-Gaussian nature can also be measured/constrained in addition to the power spectrum $\# 1$.

Compared to the counterpart of adiabatic fluctuations, observational constraints on isocurvature non-Gaussianity using actual data have almost not been studied ${ }^{\# 2}$, except a few works [24,25]. In Ref. [25], we have obtained observational constraints on isocurvature non-Gaussianity by adopting an optimal bispectrum estimator, focusing on uncorrelated type of CDM (baryon) mode. The constraints would give interesting implications to particle physics as well since this type of isocurvature fluctuations can be generated in some models such as the axion model [26-33] and Affleck-Dine baryogenesis [34-36].

In fact, isocurvature fluctuations can also be correlated with adiabatic ones in some cases and such correlated type is naturally produced in the curvaton model [37-39], which has been attracting attention due to its possibility of getting large non-Gaussianity. In the model, depending on when and how DM or baryon asymmetry are produced, positively and negatively correlated isocurvature fluctuations can be generated [19,40-51]. Furthermore, isocurvature fluctuations can also be associated with neutrino [52], which generates neutrino density isocurvature mode ${ }^{\# 3}$. Such a neutrino mode can be related to lepton asymmetry, thus its constraints may give interesting implications to the lepton sector.

In the light of these considerations, it would be also worth investigating observational constraints on isocurvature non-Gaussianities of correlated type and/or other modes in addition to CDM (baryon) one such as the neutrino mode mentioned above. The main purpose of this paper is to study the constraints on non-Gaussianity of those kinds of isocurvature types/modes by extending the analysis of our previous study [25]. By using the optimal estimator which we adopted there, we investigate observational constraints on non-linearity parameters for correlated/uncorrelated CDM and neutrino isocurvature modes from WMAP7 data. Implications of our results are also discussed for some explicit

\footnotetext{
\#1 Future observations of redshifted $21 \mathrm{~cm}$ line emission can also probe the isocurvature perturbations $[11,12]$.

\#2 See Refs. [13-16] for expected constraints from future observations by using Fisher matrix analysis. For theoretical aspects of isocurvature non-Gaussianity, see Refs. [16-23].

\#3 There is also another isocurvature mode associated with neutrino called neutrino isocurvature velocity (NIV) mode [52], in which there is difference between initial velocity perturbations of neutrino and photon. However, there are so far no concrete models which can generate NIV perturbations at super-horizon scales. We do not study NIV mode in this paper.
} 
model.

The organization of this paper is as follows. In Section 2, we present the models of non-Gaussian isocurvature perturbations which we are to constrain in this paper and their CMB bispectra. We also make the Fisher matrix analysis to get some idea for the size of the error, which also serves as a check of our results presented in Section 4. Implications of our constraints are discussed for the curvaton scenario in Section 5. The final section is devoted to conclusion.

In this paper, we denote the mean value and perturbation of a fluctuating field by bar and delta, respectively. A bracket \langle\rangle represents an ensemble average.

\section{Non-Gaussian primordial perturbations and CMB bispectrum}

\subsection{Non-Gaussian perturbations}

Following mostly Refs. [22,23], here we briefly summarize the formalism to discuss nonGaussianities of primordial curvature and isocurvature perturbations. Based on the $\delta N$ formalism [53-56], the curvature perturbation on the uniform density hypersurface of a component $i$, which we denote $\zeta_{i}$, can be given by

$$
\zeta_{i}(\vec{x}, t)=N_{i}(\vec{x}, t)-\bar{N}_{i}(t)
$$

where $N(\vec{x}, t)$ is an $e$-folding number from the initial flat hypersurface to the uniform density hypersurface of $i$ at time $t$. In particular, we denote the curvature perturbation on the uniform density hypersurface of the total matter as $\zeta_{\text {tot }}$. Then we adopt the following definition of the curvature $\zeta$ and isocurvature perturbations $S_{i}$,

$$
\begin{aligned}
\zeta(\vec{x}, t) & \equiv \zeta_{\text {tot }}(\vec{x}, t), \\
S_{i}(\vec{x}, t) & \equiv 3\left\{\zeta_{i}(\vec{x}, t)-\zeta_{\text {tot }}(\vec{x}, t)\right\} .
\end{aligned}
$$

In this paper, we consider two types of primordial isocurvature perturbations, which are CDM one (CI) and neutrino density one (NID).

In the picture of the separate Universe, the spatial dependence of $N_{i}(\vec{x}, t)$ should come only from the initial values of the degrees of freedom (or scalar fields) during inflation, which we write as $\phi_{a}(\vec{x})=\bar{\phi}_{a}+\delta \phi_{a}(\vec{x})$ with a subscript a represents a species of scalar fields. We can expand the curvature and isocurvature perturbations $\zeta$ and $S_{i}$ locally in terms of $\phi$ up to second order, which leads to

$$
\begin{aligned}
\zeta(\vec{x}, t) & =N_{\mathrm{tot}, a}(t) \delta \phi_{a}(\vec{x})+\frac{1}{2} N_{\mathrm{tot}, a b}(t)\left\{\delta \phi_{a}(\vec{x}) \delta \phi_{b}(\vec{x})-\left\langle\delta \phi_{a}(\vec{x}) \delta \phi_{b}(\vec{x})\right\rangle\right\}+\cdots, \\
S_{i}(\vec{x}, t) & =S_{i, a}(t) \delta \phi_{a}(\vec{x})+\frac{1}{2} S_{i, a b}(t)\left\{\delta \phi_{a}(\vec{x}) \delta \phi_{b}(\vec{x})-\left\langle\delta \phi_{a}(\vec{x}) \delta \phi_{b}(\vec{x})\right\rangle\right\}+\cdots,
\end{aligned}
$$


where the coefficients with subscripts $a, b$, etc. attached to $N$ represent derivatives with respect to the initial fields, e.g.,

$$
N_{\mathrm{tot}, a}(t)=\left.\frac{\partial N_{\mathrm{tot}}(\vec{x}, t)}{\partial \phi_{a}(\vec{x})}\right|_{\phi(\vec{x})=\bar{\phi}} .
$$

In this paper, we assume that the initial fluctuations $\delta \phi_{a}$ are Gaussian and different initial fields are uncorrelated, i.e. $\left\langle\delta \phi_{a} \delta \phi_{b}\right\rangle=0$ for $a \neq b$. Nonlinearity parameters of the local type for the curvature and isocurvature perturbations are defined as follows:

$$
\begin{aligned}
\zeta(\vec{x}) & =\zeta_{\mathrm{G}}(\vec{x})+\frac{3}{5} f_{\mathrm{NL}}\left(\zeta_{\mathrm{G}}^{2}(\vec{x})-\left\langle\zeta_{\mathrm{G}}^{2}(\vec{x})\right\rangle\right), \\
S_{i}(\vec{x}) & =S_{i \mathrm{G}}(\vec{x})+f_{\mathrm{NL}}^{(\mathrm{ISO})}\left(S_{i \mathrm{G}}^{2}(\vec{x})-\left\langle S_{i \mathrm{G}}^{2}(\vec{x})\right\rangle\right),
\end{aligned}
$$

where $\zeta_{\mathrm{G}}$ and $S_{i \mathrm{G}}$ are the Gaussian part of $\zeta$ and $S_{i}$, respectively. Eqs. (7) and (8) can be recast into a general form:

$$
X^{A}(\vec{x})=X_{\mathrm{G}}^{A}(\vec{x})+f_{\mathrm{NL}}^{A}\left(X_{\mathrm{G}}^{A}(\vec{x})^{2}-\left\langle X_{\mathrm{G}}^{A}(\vec{x})^{2}\right\rangle\right),
$$

where $X_{\mathrm{G}}^{A}$ is the Gaussian part of $X^{A}$. Here we defined a non-linearity parameter $f_{\mathrm{NL}}^{A}$, which is related to the adiabatic and isocurvature ones via $f_{\mathrm{NL}}^{\zeta}=\frac{3}{5} f_{\mathrm{NL}}$ and $f_{\mathrm{NL}}^{S}=f_{\mathrm{NL}}^{(\mathrm{ISO})}$.

We present constraints in two limiting cases, both of which are of particular interest. In one limiting case, $\zeta_{\mathrm{G}}$ and $S_{i \mathrm{G}}$ are assumed to be uncorrelated, i.e. $\left\langle\zeta_{\mathrm{G}} S_{i \mathrm{G}}\right\rangle=0$. This is the case that we discussed for CDM (baryon) isocurvature mode in the previous paper [25]. In terms of the scalar field fluctuations, this corresponds to the case that the curvature and isocurvature perturbations are generated from two different fields $\phi$ and $\sigma$, i.e. $\zeta_{\mathrm{G}} \propto \delta \phi$ and $S_{i \mathrm{G}} \propto \delta \sigma$. As we have stated in Introduction, this form of non-Gaussian isocurvature perturbations can be generated in e.g. the axion model [26-33] and the Affleck-Dine mechanism [34-36]. In another limiting case, $\zeta_{\mathrm{G}}$ and $S_{i \mathrm{G}}$ are assumed to be totally (anti-) correlated, so that $\left\langle\zeta_{\mathrm{G}} S_{i \mathrm{G}}\right\rangle= \pm \sqrt{\left\langle\zeta_{\mathrm{G}} \zeta_{\mathrm{G}}\right\rangle\left\langle S_{i \mathrm{G}} S_{i \mathrm{G}}\right\rangle}$. This corresponds to the cases where $\zeta$ and $S_{i \mathrm{G}}$ are generated from only a single field $\phi$, so that $\zeta_{\mathrm{G}} \propto S_{i \mathrm{G}} \propto \delta \phi$. Such a case can be realized in e.g. the curvaton scenario $[41,43]$.

From Eq. (9), the primordial bispectrum can be given by

$$
\left\langle X^{A}\left(\vec{k}_{1}\right) X^{B}\left(\vec{k}_{2}\right) X^{C}\left(\vec{k}_{3}\right)\right\rangle=\left[f_{\mathrm{NL}}^{A} P_{A B}\left(k_{2}\right) P_{A C}\left(k_{3}\right)+(2 \text { perms })\right](2 \pi)^{3} \delta^{(3)}\left(\vec{k}_{1}+\vec{k}_{2}+\vec{k}_{3}\right),
$$

where $P_{A B}(k)$ is the power spectrum of (cross-)correlation between $X_{\mathrm{G}}^{A}$ and $X_{\mathrm{G}}^{B}$, i.e.

$$
\left\langle X_{\mathrm{G}}^{A}(\vec{k}) X_{\mathrm{G}}^{B}\left(\vec{k}^{\prime}\right)\right\rangle \equiv P_{A B}(k)(2 \pi)^{3} \delta\left(\vec{k}+\vec{k}^{\prime}\right) .
$$

Note that in Eq. (10), we omitted the loop contributions, assuming that the tree terms are leading ones in the power spectrum and the non-linearity parameters.

Current observations have not detected any isocurvature perturbations and only the upper bounds on the amplitudes of the isocurvature power spectra have been provided. 
The upper bounds depend on the kinds of isocurvature modes and their correlations with the curvature perturbations. We define the ratio of the amplitude of isocurvature power spectrum to that of adiabatic one at the reference scale $k_{*}$ as

$$
\alpha \equiv \frac{P_{S_{i} S_{i}}\left(k_{*}\right)}{P_{\zeta \zeta}\left(k_{*}\right)} .
$$

In this paper, we take the WMAP convention $k_{*}=0.002 \mathrm{Mpc}^{-1}$. In the case of CI, WMAP 7 -year result [58] gives upper bounds $\# 4$

$$
\begin{aligned}
& \alpha<0.15 \quad \text { (uncorrelated), } \\
& \alpha<0.008 \quad \text { (totally correlated). }
\end{aligned}
$$

For the case of NID, a combined analysis from the WMAP 7-year and the ACT data [59] gives upper bounds $[60]^{\# 5}$

$$
\begin{aligned}
& \alpha<0.19 \quad \text { (uncorrelated), } \\
& \alpha<0.013 \quad \text { (totally correlated). }
\end{aligned}
$$

Here some comments on the parametrization of non-linearity parameters are in order. When we consider the bispectrum with multiple sources, one can generally define the non-linearity parameters $\tilde{f}_{\mathrm{NL}}^{A, B C}$ as follows [14]:

$$
\begin{aligned}
\left\langle X^{A}\left(\vec{k}_{1}\right) X^{B}\left(\vec{k}_{2}\right) X^{C}\left(\vec{k}_{3}\right)\right\rangle & =(2 \pi)^{3} \delta^{(3)}\left(\vec{k}_{1}+\vec{k}_{2}+\vec{k}_{3}\right) \\
& \times\left[\tilde{f}_{\mathrm{NL}}^{A, B C} P_{\zeta}\left(k_{2}\right) P_{\zeta}\left(k_{3}\right)+\tilde{f}_{\mathrm{NL}}^{B, C A} P_{\zeta}\left(k_{2}\right) P_{\zeta}\left(k_{3}\right)+\tilde{f}_{\mathrm{NL}}^{C, A B} P_{\zeta}\left(k_{2}\right) P_{\zeta}\left(k_{3}\right)\right],
\end{aligned}
$$

where $\tilde{f}_{\mathrm{NL}}^{A, B C}=\tilde{f}_{\mathrm{NL}}^{A, C B}$ is satisfied due to the symmetric structure for the latter two indices and the (total) curvature power spectrum is used instead of $P_{A B}$ defined in Eq. (11). When we consider the case, where curvature and isocurvature perturbations (of a single type) coexist, there exist 6 non-linearity parameters in general: $\tilde{f}_{\mathrm{NL}}^{\zeta, \zeta \zeta}, \tilde{f}_{\mathrm{NL}}^{\zeta, \zeta S}, \tilde{f}_{\mathrm{NL}}^{\zeta, S S}, \tilde{f}_{\mathrm{NL}}^{S, \zeta \zeta}, \tilde{f}_{\mathrm{NL}}^{S, S \zeta}, \tilde{f}_{\mathrm{NL}}^{S, S S}$. However, if one considers uncorrelated or totally correlated isocurvature models, these parameters can be reduced or related in some way. By using $f_{\mathrm{NL}}^{\zeta}=\frac{3}{5} f_{\mathrm{NL}}$ and $f_{\mathrm{NL}}^{S}=f_{\mathrm{NL}}^{(\mathrm{ISO})}$ defined in Eqs. (7) and (8), for uncorrelated models, the above non-linearity parameters are given by

$$
\tilde{f}_{\mathrm{NL}}^{\zeta, \zeta \zeta}=f_{\mathrm{NL}}^{\zeta}, \quad \tilde{f}_{\mathrm{NL}}^{S, S S}=\alpha^{2} f_{\mathrm{NL}}^{S}, \quad \tilde{f}_{\mathrm{NL}}^{A, B C}=0 \quad(\text { others }) .
$$

\footnotetext{
\#4 In Ref. [58], only the positively-correlated CI model is studied, and does not investigate the negativelycorrelated one. However, since $\alpha$ allowed observationally is so small, that the upper bounds on $\alpha$ for totally correlated cases would not depend much on the sign of the correlation.

\#5 We note that the effective number of neutrinos $N_{\text {eff }}$ is also varied in Ref. [60]. However, the degeneracy between $\alpha$ and $N_{\text {eff }}$ is not severe, thus we expect that constraints with fixed $N_{\text {eff }}=3$ should be almost the same. For most recent constraints on $\alpha$ with fixed $N_{\text {eff }}$, we refer to Ref. [1], which uses the WMAP 3 -year data.
} 
For totally correlated models, we have

$$
\begin{array}{rll}
\tilde{f}_{\mathrm{NL}}^{\zeta, \zeta \zeta}=f_{\mathrm{NL}}^{\zeta}, & \tilde{f}_{\mathrm{NL}}^{\zeta, \zeta S}= \pm \alpha^{1 / 2} f_{\mathrm{NL}}^{\zeta}, & \tilde{f}_{\mathrm{NL}}^{\zeta, S S}=\alpha f_{\mathrm{NL}}^{\zeta}, \\
\tilde{f}_{\mathrm{NL}}^{S, \zeta \zeta}=\alpha f_{\mathrm{NL}}^{S}, & \tilde{f}_{\mathrm{NL}}^{S, \zeta S}= \pm \alpha^{3 / 2} f_{\mathrm{NL}}^{S}, & \tilde{f}_{\mathrm{NL}}^{S, S S}=\alpha^{2} f_{\mathrm{NL}}^{S},
\end{array}
$$

where the signs should be $+/$ - if isocurvature perturbations are positively/negatively correlated with curvature ones.

Thus, for uncorrelated modes, 6 parameters reduces to $f_{\mathrm{NL}}^{\zeta}$ and the combination of $\alpha^{2} f_{\mathrm{NL}}^{S}$, which we investigate in the analysis of this paper. For correlated modes, we still need 6 parameters. Although in principle, we can probe all of these parameters/combinations, we focus on $f_{\mathrm{NL}}^{\zeta}=\tilde{f}_{\mathrm{NL}}^{\zeta, \zeta \zeta}$ and $\alpha f_{\mathrm{NL}}^{S}=\tilde{f}_{\mathrm{NL}}^{S, \zeta \zeta}$ for the correlated case since the contributions to the bispectrum from other isocurvature modes are weak, hence the

constraint on $\tilde{f}_{\mathrm{NL}}^{\zeta, \zeta \zeta}$ and $\tilde{f}_{\mathrm{NL}}^{S, \zeta \zeta}$ would be most severe. We will again discuss this issue after giving a summary of CMB bispectrum in the next subsection.

\subsection{CMB bispectrum}

Now we briefly summarize the formalism to calculate CMB bispectrum. In the analysis, we neglect the secondary non-Gaussianities arising from the second or higher order cosmological perturbation theory.

The harmonic coefficients of the CMB temperature anisotropy from primordial perturbations $X^{A}$ can be given as

$$
a_{l m}^{A}=4 \pi(-i)^{l} \int \frac{d k^{3}}{(2 \pi)^{3}} g_{l}^{A}(k) X^{A}(\vec{k}) Y_{l m}^{*}(\hat{k}),
$$

where $g_{l}^{A}(k)$ is the temperature transfer function for the primordial perturbations $X^{A}$. The total CMB anisotropy is the sum of those from the curvature and isocurvature perturbations, i.e. $a_{l m}=a_{l m}^{\zeta}+a_{l m}^{S}$. The angular power spectrum $C_{l}$, defined by $\left\langle a_{l m} a_{l^{\prime} m^{\prime}}{ }^{*}\right\rangle=$ $C_{l} \delta_{l l^{\prime}} \delta_{m m^{\prime}}$, can be written as

$$
C_{l}=\frac{2}{\pi} \sum_{A B} \int d k k^{2} g_{l}^{A}(k) g_{l}^{B}(k) P_{A B}(k) .
$$

It is convenient to define the reduced bispectrum $b_{l_{1} l_{2} l_{3}}$ as

$$
\left\langle a_{l_{1} m_{1}} a_{l_{2} m_{2}} a_{l_{3} m_{3}}\right\rangle=b_{l_{1} l_{2} l_{3}} \mathcal{G}_{m_{1} m_{2} m_{3}}^{l_{1} l_{2} l_{3}},
$$

where $\mathcal{G}_{m_{1} m_{2} m_{3}}^{l_{1} l_{2} l_{3}}$ is the Gaunt integral, which can be written in terms of the Wigner-3j symbol as

$$
\mathcal{G}_{m_{1} m_{2} m_{3}}^{l_{1} l_{2} l_{3}}=\sqrt{\frac{\left(2 l_{1}+1\right)\left(2 l_{2}+1\right)\left(2 l_{3}+1\right)}{4 \pi}}\left(\begin{array}{ccc}
l_{1} & l_{2} & l_{3} \\
m_{1} & m_{2} & m_{3}
\end{array}\right)\left(\begin{array}{ccc}
l_{1} & l_{2} & l_{3} \\
0 & 0 & 0
\end{array}\right) .
$$


Given the bispectrum of primordial perturbations in Eq. (10), the reduced CMB bispectrum is

$$
b_{l_{1} l_{2} l_{3}}=2 \sum_{A B C} f_{\mathrm{NL}}^{A} \int d r r^{2}\left(\alpha_{l_{1}}^{A}(r) \beta_{l_{2}}^{B, A}(r) \beta_{l_{3}}^{C, A}(r)+(2 \text { perms })\right),
$$

where $\alpha_{l}^{A}(r)$ and $\beta_{l}^{B, A}(r)$ are

$$
\begin{aligned}
\alpha_{l}^{A}(r) & =\frac{2}{\pi} \int d k k^{2} g_{l}^{A}(k) j_{l}(k r), \\
\beta_{l}^{B, A}(r) & =\frac{2}{\pi} \int d k k^{2} g_{l}^{B}(k) j_{l}(k r) P_{A B}(k) .
\end{aligned}
$$

By defining

$$
b_{l_{1} l_{2} l_{3}}^{A, B C}=2 \int d r r^{2}\left(\alpha_{l_{1}}^{A}(r) \beta_{l_{2}}^{B, A}(r) \beta_{l_{3}}^{C, A}(r)+(2 \text { perms })\right),
$$

Eq. (24) can be recast into

$$
b_{l_{1} l_{2} l_{3}}=\sum_{A B C}\left[f_{\mathrm{NL}}^{A} b_{l_{1} l_{2} l_{3}}^{A, B C}+(2 \text { perms })\right]
$$

For later convenience, we introduce a normalized bispectrum

$$
\begin{aligned}
\hat{b}_{l_{1} l_{2} l_{3}}^{A} & \equiv \frac{\partial b_{l_{1} l_{2} l_{3}}}{\partial f_{\mathrm{NL}}^{A}} \\
& =\sum_{B C}\left[b_{l_{1} l_{2} l_{3}}^{A, B C}+(2 \text { perms })\right]
\end{aligned}
$$

with which we can rewrite Eq. (28) as

$$
b_{l_{1} l_{2} l_{3}}=\sum_{A} f_{\mathrm{NL}}^{A} \hat{b}_{l_{1} l_{2} l_{3}}^{A}
$$

When we consider primordial perturbations of uncorrelated isocurvature type, we obtain

$$
\begin{aligned}
& \hat{b}_{l_{1} l_{2} l_{3}}^{\zeta}=b_{l_{1} l_{2} l_{3}}^{\zeta, \zeta \zeta}, \\
& \hat{b}_{l_{1} l_{2} l_{3}}^{S}=b_{l_{1} l_{2} l_{3}}^{S, S S} .
\end{aligned}
$$

Since $\hat{b}_{l_{1} l_{2} l_{3}}^{S} \propto \alpha^{2} f_{\mathrm{NL}}^{S}$, CMB anisotropy is affected by the combination of $\alpha^{2} f_{\mathrm{NL}}^{S}$. Thus what we can constrain from CMB experiments is $\alpha^{2} f_{\mathrm{NL}}^{S}$, not $f_{\mathrm{NL}}^{S}$ alone.

For the case of correlated isocurvature perturbations, we obtain

$$
\begin{aligned}
& \hat{b}_{l_{1} l_{2} l_{3}}^{\zeta}=b_{l_{1} l_{2} l_{3}}^{\zeta, \zeta \zeta}+2 b_{l_{1} l_{2} l_{3}}^{\zeta, \zeta S}+b_{l_{1} l_{2} l_{3}}^{\zeta, S S}, \\
& \hat{b}_{l_{1} l_{2} l_{3}}^{S}=b_{l_{1} l_{2} l_{3}}^{S, \zeta \zeta}+2 b_{l_{1} l_{2} l_{3}}^{S, \zeta S}+b_{l_{1} l_{2} l_{3}}^{S, S S} .
\end{aligned}
$$


In terms of $\alpha$, the second and third terms in the right hand side in each equation are respectively of $\mathcal{O}(\sqrt{\alpha})$ and $\mathcal{O}(\alpha)$ higher order than the first terms. From current observations, $\alpha$ should be small as $\alpha \lesssim 0.01$, hence the first terms in Eqs. (33) and (34) are leading in the total bispectrum of Eq. (30), which are proportional to $f_{\mathrm{NL}}^{\zeta}$ and $\alpha f_{\mathrm{NL}}^{S}$, respectively. Thus the constraints on these quantities are most severe among the non-linearity parameters/combinations listed in Eq. (19). However, as mentioned in the previous subsection, observations are in principle also sensitive to the amplitudes of $b_{l_{1} l_{2} l_{3}}^{\zeta, \zeta S}, b_{l_{1} l_{2} l_{3}}^{\zeta, S S}, b_{l_{1} l_{2} l_{3}}^{S, \zeta S}$ and $b_{l_{1} l_{2} l_{3}}^{S, S S}$, which are respectively proportional to $\alpha^{1 / 2} f_{\mathrm{NL}}^{\zeta}, \alpha f_{\mathrm{NL}}^{\zeta}, \alpha^{3 / 2} f_{\mathrm{NL}}^{S}$ and $\alpha^{2} f_{\mathrm{NL}}^{S}$. Although their contribution could also affect constraints on $f_{\mathrm{NL}}$ and $\alpha f_{\mathrm{NL}}^{(\mathrm{ISO})}$, nevertheless, such an effect is expected to be weak as far as $\alpha$ is small as $\alpha \lesssim 0.01$. We will come back to this issue at the end of Section 4.

\section{$2.3 \quad$ Fisher matrix analysis}

Before presenting our constraints from observed data, we here present expected errors from WMAP7 data based on the Fisher matrix analysis, which serves as a cross-check of our results given in Section 4.

According to Refs. [61,62], given the bispectrum of Eq. (30), the Fisher matrix for the bispectrum is given by

$$
F_{A B}=\frac{f_{\text {sky }}}{6} \sum_{l_{\max } \geq l_{1}, l_{2}, l_{3} \geq 2} \frac{\left(2 l_{1}+1\right)\left(2 l_{2}+1\right)\left(2 l_{3}+1\right)}{4 \pi}\left(\begin{array}{ccc}
l_{1} & l_{2} & l_{3} \\
0 & 0 & 0
\end{array}\right)^{2} \frac{\hat{b}_{l_{1} l_{2} l_{3}}^{A} \hat{b}_{l_{1} l_{2} l_{3}}^{B}}{\mathcal{C}_{l_{1}} \mathcal{C}_{l_{2}} \mathcal{C}_{l_{3}}},
$$

where $\mathcal{C}_{l}=C_{l}+N_{l}$ with $C_{l}$ and $N_{l}$ being the power spectra of signal and noise, respectively. Given a survey with a finite resolution, $F_{A B}$ saturates as $l_{\max }$ are increased.

The signal power spectrum $C_{l}$ are computed using the CAMB code [63] assuming the mean parameters from the WMAP 7-year data alone [58]. We set the same spectral indices for both the power spectra of adiabatic and isocurvature perturbations. On the other hand, the noise power spectrum can be approximated with the Knox's formula [64] as,

$$
N_{l}=\theta_{\mathrm{FWHM}}^{2} \sigma_{T}^{2} \exp \left[l(l+1) \frac{\theta_{\mathrm{FWHM}}^{2}}{8 \ln 2}\right]
$$

where $\theta_{\mathrm{FWHM}}$ is the full width at half maximum of the Gaussian beam, and $\sigma_{T}$ is the root mean square of the instrumental noise par pixel. The total $N_{l}$ of a multi-frequency survey can be given by a quadrature sum of $N_{l}$ of each frequency band. For the Fisher matrix analysis, we adopt the specification of the 7-year WMAP V and W bands for $\theta_{\mathrm{FWHM}}$ and $\sigma_{T}$, which are are listed in Table 1 [65]. Furthermore, we assume that $f_{\text {sky }}=0.72$, which is consistent with the mask we adopt in Section 4.

From $F_{A B}$, we can evaluate ideal constraints on the nonlinearity parameters. For a single nonlinearity parameter, two different constraints can be derived. One is the nonmarginalized constraint in which we assume that the other nonlinearity parameter is fixed to zero. In this case, $1 \sigma$ error should be given by $\Delta f_{\mathrm{NL}}^{A}=1 / \sqrt{F_{A A}}$. Another one is the 


\begin{tabular}{llc}
\hline \hline band & $\mathrm{V}$ & $\mathrm{W}$ \\
\hline$\theta_{\text {FWHM }}[\operatorname{arcmin}]$ & 21.0 & 13.2 \\
$\sigma_{T}[\mathrm{mK}]$ & 25 & 38 \\
\hline \hline
\end{tabular}

Table 1: Survey parameters for 7-year observation of the WMAP V and W bands.

marginalized constraint which assumes the other nonlinearity parameter varies freely. In this case, $1 \sigma$ error is given by $\Delta f_{\mathrm{NL}}^{A}=\sqrt{F_{A A}^{-1}}$. In Table 2 , we list ideal $1 \sigma$ constraints from the WMAP 7-year observation for uncorrelated CI and NID models. Those for the correlated CI and NID models are listed in Table 3. These results are to be consulted for validity check of our constraints from actual data in Section 4.

\begin{tabular}{llc}
\hline \hline & $\Delta f_{\mathrm{NL}}$ & $\Delta\left(\alpha^{2} f_{\mathrm{NL}}^{(\mathrm{ISO})}\right)$ \\
\hline uncorrelated CI & $20(22)$ & $65(72)$ \\
uncorrelated NID & $20(38)$ & $135(256)$ \\
\hline \hline
\end{tabular}

Table 2: $1 \sigma$ errors evaluated from the Fisher matrix analysis on the nonlinearity parameters from the WMAP 7-year observation for the uncorrelated CI and NID models. Errors with and without parenthesis correspond to non-marginalized and marginalized constraints, respectively.

\begin{tabular}{llc}
\hline \hline & $\Delta f_{\mathrm{NL}}$ & $\Delta\left(\alpha f_{\mathrm{NL}}^{(\mathrm{ISO})}\right)$ \\
\hline correlated CI & $20(24)$ & $120(140)$ \\
correlated NID & $20(56)$ & $51(143)$ \\
\hline \hline
\end{tabular}

Table 3: The same as in Table 2 except for the correlated CI and NID models.

\section{Analysis method}

Our analysis method is the same as in our previous paper [25]. For more details, we refer to Ref. [25] and references therein.

We adopt the following optimal estimator of nonlinearity parameters $f_{\mathrm{NL}}^{A}$,

$$
\left(\begin{array}{c}
\hat{f}_{\mathrm{NL}}^{\zeta} \\
\hat{f}_{\mathrm{NL}}^{S}
\end{array}\right)=\left(\begin{array}{ll}
\left\langle S_{\text {prim }}^{\zeta}\right\rangle_{f_{\mathrm{NL}}^{\zeta}=1} & \left\langle S_{\text {prim }}^{\zeta}\right\rangle_{f_{\mathrm{NL}}^{S}=1}^{S} \\
\left\langle S_{\text {prim }}^{S}\right\rangle_{f_{\mathrm{NL}}^{\zeta}=1}^{\zeta} & \left\langle S_{\text {prim }}^{S}\right\rangle_{f_{\mathrm{NL}}^{S}=1}^{S}
\end{array}\right)^{-1}\left(\begin{array}{c}
S_{\text {prim }}^{\zeta} \\
S_{\text {prim }}^{S}
\end{array}\right),
$$


where a square bracket with subscript $f_{\mathrm{NL}}^{A}=1$ indicates an ensemble average over simulations with non-zero non-linearity parameter shown in the subscript and the other nonlinearity parameter being fixed to zero. $S_{\text {prim }}^{A}$ is the cubic statistics [66-69], which is given by

$$
S_{\text {prim }}^{A}=\frac{1}{6} \sum_{\{l m\}} \hat{b}_{l_{1} l_{2} l_{3}}^{A} \mathcal{G}_{m_{1} m_{2} m_{3}}^{l_{1} l_{2} l_{3}}\left[\tilde{a}_{l_{1} m_{1}} \tilde{a}_{l_{2} m_{2}} \tilde{a}_{l_{3} m_{3}}-3 \tilde{a}_{l_{1} m_{1}}\left\langle\tilde{a}_{l_{2} m_{2}} \tilde{a}_{l_{3} m_{3}}\right\rangle_{0}\right],
$$

where the square bracket with subscript 0 indicates an ensemble average over Gaussian simulations. $\tilde{a}_{l m}$ is a harmonic coefficient of observed (or simulated) data maps weighted by inverse-variance,

$$
\tilde{a}_{l m} \equiv \sum_{l^{\prime} m^{\prime}} \mathcal{C}_{l m, l^{\prime} m^{\prime}}^{-1} a_{l^{\prime} m^{\prime}}
$$

which is derived from the optimality of the estimator of Eq. (37) [70]. Eq. (37) can be schematically represented as $\hat{f}_{\mathrm{NL}}^{A}=\sum_{B}\left\langle S_{\text {prim }}^{A}\right\rangle_{f_{\mathrm{NL}}^{B}=1}^{-1} S_{\text {prim }}^{B}$. In our analysis, to evaluate $\left\langle\tilde{a}_{l m} \tilde{a}_{l^{\prime} m^{\prime}}\right\rangle_{0}$, we accumulated at least 250 Monte Carlo (MC) samples.

Assuming the Gaussianity for $X^{A}$, we estimate the covariance of the estimator $\hat{f}_{\mathrm{NL}}^{A}$,

$$
\begin{aligned}
\left\langle\hat{f}_{\mathrm{NL}}^{A} \hat{f}_{\mathrm{NL}}^{B}\right\rangle_{0} & =\sum_{A^{\prime} B^{\prime}}\left\langle S_{\text {prim }}^{A}\right\rangle_{f_{\mathrm{NL}}^{A^{\prime}}=1}^{-1}\left\langle S_{\text {prim }}^{A^{\prime}} S_{\text {prim }}^{B^{\prime}}\right\rangle_{0}\left\langle S_{\text {prim }}^{B}\right\rangle_{f_{\mathrm{NL}}^{B^{\prime}}=1}^{-1} \\
& =\left\langle S_{\mathrm{prim}}^{A}\right\rangle_{f_{\mathrm{NL}}^{B}=1}^{-1} .
\end{aligned}
$$

Here we used the relation $\left\langle S_{\text {prim }}^{A}\right\rangle_{f_{\mathrm{NL}}^{B}=1}=\left\langle S_{\text {prim }}^{A} S_{\text {prim }}^{B}\right\rangle_{0}$, which has been proved in our previous paper [25]. When we assume that either of adiabatic or uncorrelated isocurvature perturbations are non-Gaussian and the other is Gaussian, Eq. (37) can be reduced to

$$
\hat{f}_{\mathrm{NL}}^{A}=S_{\text {prim }}^{A} /\left\langle S_{\text {prim }}^{A}\right\rangle_{f_{\mathrm{NL}}^{A}=1}
$$

where the subscripts $A$ indicates the perturbations which are assumed to be non-Gaussian. In this case, the variance of $\hat{f}_{\mathrm{NL}}^{A}$ is given by $1 /\left\langle S_{\mathrm{prim}}^{A}\right\rangle_{f_{\mathrm{NL}}^{A}=1}$.

In order to determine the normalization $\left\langle S_{\text {prim }}^{A}\right\rangle_{f_{\mathrm{NL}}^{B}=1}$, we need to simulate non-Gaussian CMB maps. We adopt the method for local-type non-Gaussianity developed in Ref. [71], in which the quadrature method with optimized nodes and weights for line of sight integral is used. In our analysis, we require the method to be accurate such that the mean square of the error is less than 0.01 at each multipole $(l m)$. For $l_{\max }=1024$, we found that this level of accuracy requires 42, 15 and 14 nodes for adiabatic, CI and NID perturbations, respectively.

Computation of inverse-variance weighted map $\tilde{a}_{l m}$ is performed based on the method of Ref. [72], which adopts a conjugate gradient method with multi-grid preconditioning. This method also allows us to marginalize over the amplitude of components which are proportional to template maps. 


\section{Result}

In our analysis, we assume a flat power-law $\Lambda$ CDM model as a fiducial one and adopt the mean values for cosmological parameters from the WMAP 7-year data alone [58]

$$
\left(\Omega_{b}, \Omega_{c}, H_{0}, \tau, A_{\text {adi }}\right)=\left(0.0448,0.220,71,0.088,2.43 \times 10^{-9}\right) .
$$

Here $\Omega_{b}$ and $\Omega_{c}$ are the density parameters for baryon and CDM, respectively. $H_{0}$ is the Hubble constant in units of $\mathrm{km} / \mathrm{sec} / \mathrm{Mpc}$ and $\tau$ is the optical depth for reionization. $A_{\text {adi }}$ is the amplitude of power spectrum of adiabatic perturbations at the reference scale $k_{*}=0.002 \mathrm{Mpc}^{-1}$. With this definition, the power spectrum can be written as $\mathcal{P}_{\zeta \zeta}(k)=$ $\left(k^{3} / 2 \pi^{2}\right) P_{\zeta \zeta}(k)=A_{\text {adi }}\left(k / k_{*}\right)^{n_{\text {adi }}-1}$, where $n_{\text {adi }}$ is the spectral index for $P_{\zeta \zeta}$. We assume that the power spectrum of isocurvature perturbations is also given in a power-law form $\mathcal{P}_{S_{\mathrm{G}} S_{\mathrm{G}}}(k)=\left(k^{3} / 2 \pi^{2}\right) P_{S_{\mathrm{G}} S_{\mathrm{G}}}(k) \propto k^{n_{\text {iso }}-1}$. With regard to the values of $n_{\text {adi }}$ and $n_{\text {iso }}$, we adopt several different settings, which will be separately presented when we show our constraints. The transfer function of CMB is computed using the CAMB code [63]. We combine the foreground-cleaned maps of $\mathrm{V}$ and $\mathrm{W}$ bands of the WMAP 7-year data $[73,74]^{\# 6}$ with a resolution $N_{\text {side }}=512$ in the HEALPix pixelization scheme $[75]^{\# 7}$. We adopt the KQ75y7 mask [74], which cuts $28.4 \%$ of the sky. We also set the maximum multipole $l_{\max }$ to 1024 in our analysis. We marginalize the amplitudes of the monopole $l=0$ and dipoles $l=1$ as default and also optionally marginalize the amplitudes of the Galactic foreground components at large angular scales using the synchrotron, free-free and dust emission templates from Ref. [74].

Constraints for the case of uncorrelated isocurvature perturbations are listed in Table 4. Concerning the spectral indices, we fix $n_{\text {adi }}$ to the WMAP 7 -year mean value 0.963 , while for $n_{\text {iso }}$, we adopt two settings, $n_{\text {iso }}=0.963$ and $n_{\text {iso }}=1$, which are separately analyzed. Joint constraints on $f_{\mathrm{NL}}=\frac{5}{3} f_{\mathrm{NL}}^{\zeta}$ and $\alpha^{2} f_{\mathrm{NL}}^{(\mathrm{ISO})}=\alpha^{2} f_{\mathrm{NL}}^{S}$ are shown in Fig. 1. First we found that the $1 \sigma$ errors are roughly the same as those obtained from the Fisher matrix analysis given in Table 2 regardless of marginalization of foregrounds and fiducial $n_{\text {iso }}$. The fiducial values of $n_{\text {iso }}$ little affect the central values for both the uncorrelated CI and NID models. While the marginalization of foregrounds can bias the central values in the uncorrelated CI model by as much as $0.5 \sigma$, the effects in the uncorrelated NID model are smaller, from which we can see that the effects of residual foregrounds are moderate. To conclude the case for the uncorrelated modes, the primordial perturbations are consistent with Gaussian ones at around $2 \sigma$ level, even if we consider uncorrelated CI or NID perturbations.

Constraints for the case of correlated isocurvature perturbations are listed in Table 4. With regard to the values of spectral indices, we adopt two different cases, $n_{\text {adi }}=n_{\text {iso }}=$ 0.963 and $n_{\text {adi }}=n_{\text {iso }}=1$ for correlated case. Joint constraints on $f_{\mathrm{NL}}=\frac{5}{3} f_{\mathrm{NL}}^{\zeta}$ and $\alpha f_{\mathrm{NL}}^{(\mathrm{ISO})}=\alpha f_{\mathrm{NL}}^{S}$ are shown in Fig. 2. We found that the resulting constraints are consistent with the Fisher matrix analysis shown in Table 3 in this case as well, although marginalized

\footnotetext{
\#6http://lambda.gsfc.nasa.gov

\#7http://healpix.jpl.nasa.gov
} 


\begin{tabular}{|c|c|c|c|}
\hline & setups & $f_{\mathrm{NL}}$ & $\alpha^{2} f_{\mathrm{NL}}^{(\mathrm{ISO})}$ \\
\hline \multirow[t]{2}{*}{$\mathrm{CI}, n_{\text {iso }}=0.963$} & $\mathrm{w} / \mathrm{o}$ template marginalization & $\begin{array}{c}43 \pm 21 \\
(50 \pm 23)\end{array}$ & $\begin{array}{c}13 \pm 66 \\
(-51 \pm 72)\end{array}$ \\
\hline & $\mathrm{w} /$ template marginalization & $\begin{array}{c}37 \pm 21 \\
(41 \pm 23)\end{array}$ & $\begin{array}{c}22 \pm 64 \\
(-28 \pm 71)\end{array}$ \\
\hline \multirow[t]{2}{*}{ CI, $n_{\text {iso }}=1$} & w/o template marginalization & $\begin{array}{c}46 \pm 21 \\
(51 \pm 23)\end{array}$ & $\begin{array}{c}26 \pm 63 \\
(-34 \pm 69)\end{array}$ \\
\hline & w/ template marginalization & $\begin{array}{c}33 \pm 21 \\
(35 \pm 23)\end{array}$ & $\begin{array}{c}30 \pm 66 \\
(-15 \pm 72)\end{array}$ \\
\hline \multirow[t]{2}{*}{ NID, $n_{\text {iso }}=0.963$} & w/o template marginalization & $\begin{array}{c}43 \pm 21 \\
(65 \pm 39)\end{array}$ & $\begin{array}{c}191 \pm 140 \\
(-173 \pm 261)\end{array}$ \\
\hline & w/ template marginalization & $\begin{array}{c}34 \pm 21 \\
(48 \pm 39)\end{array}$ & $\begin{array}{c}164 \pm 143 \\
(-116 \pm 266)\end{array}$ \\
\hline \multirow[t]{2}{*}{ NID, $n_{\text {iso }}=1$} & w/o template marginalization & $\begin{array}{c}40 \pm 21 \\
(57 \pm 40)\end{array}$ & $\begin{array}{c}178 \pm 137 \\
(-133 \pm 257)\end{array}$ \\
\hline & w/ template marginalization & $\begin{array}{c}36 \pm 21 \\
(48 \pm 40)\end{array}$ & $\begin{array}{c}175 \pm 137 \\
(-87 \pm 257)\end{array}$ \\
\hline
\end{tabular}

Table 4: Constraints on $f_{\mathrm{NL}}$ and $\alpha^{2} f_{\mathrm{NL}}^{(\mathrm{ISO})}$ at $1 \sigma$ level for the cases of uncorrelated isocurvature perturbations. A value with (without) parenthesis is a constraint on a nonlinearity parameter without (with) marginalization of the other one.

errors for the NID model are substantially larger than the Fisher analysis, which may result from the inhomogeneous noise and the sky cut. We found that fiducial values of the spectral indices little affect the constraints. On the other hand, template marginalization can bias the central values by as much as $0.5 \sigma$, in particular for CI model. However, as in the uncorrelated case, the effects of residual foregrounds are regarded not to be severe at all. We can conclude that the primordial perturbations are consistent with Gaussian ones at around $2 \sigma$ level also in the case of correlated CI and NID models.

Finally we comment on the contributions from the terms which are higher order with $\mathcal{O}(\sqrt{\alpha})$ and $\mathcal{O}(\alpha)$ such as the second and third terms in Eqs. (33) and (34). In the analysis of correlated isocurvature models, we have neglected those terms since they are expected to give little effects on the constraints. To check this, we have also made an analysis where we included those terms. We have compared the constraints with and without those terms for the case of $\alpha=0.003$. It is found that the errors are almost unchanged and the central values change by no more than $\mathcal{O}(0.1) \sigma$, which justifies ignoring higher order terms in $\alpha$ in our analysis. 

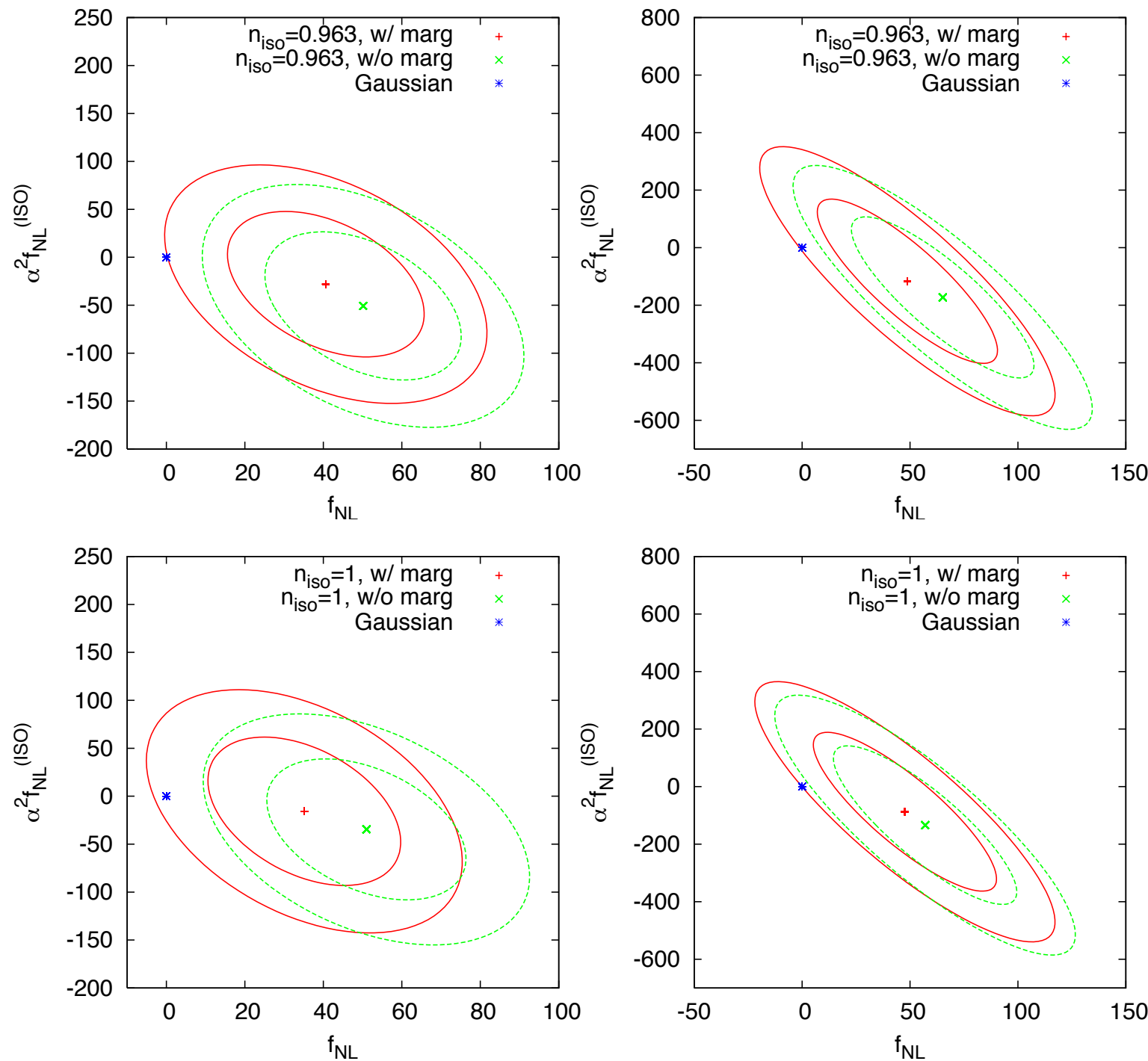

Figure 1: Joint constraints on $f_{\mathrm{NL}}$ and $\alpha^{2} f_{\mathrm{NL}}^{(\mathrm{ISO})}$ at 1 and $2 \sigma$ levels for uncorrelated isocurvature models. Different panels show cases for different isocurvature modes and

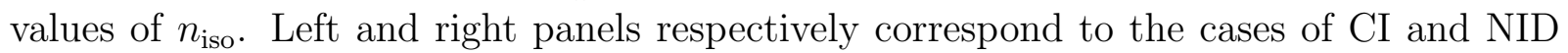
modes. Top and bottom panels respectively correspond to the cases of $n_{\text {iso }}=0.963$ and $n_{\text {iso }}=1$. Here $n_{\text {adi }}$ is fixed to 0.963 . In each panel, solid red (green dashed) lines are the constraints with (without) template marginalization of foregrounds. Blue star corresponds to the case where primordial perturbations are purely Gaussian. 

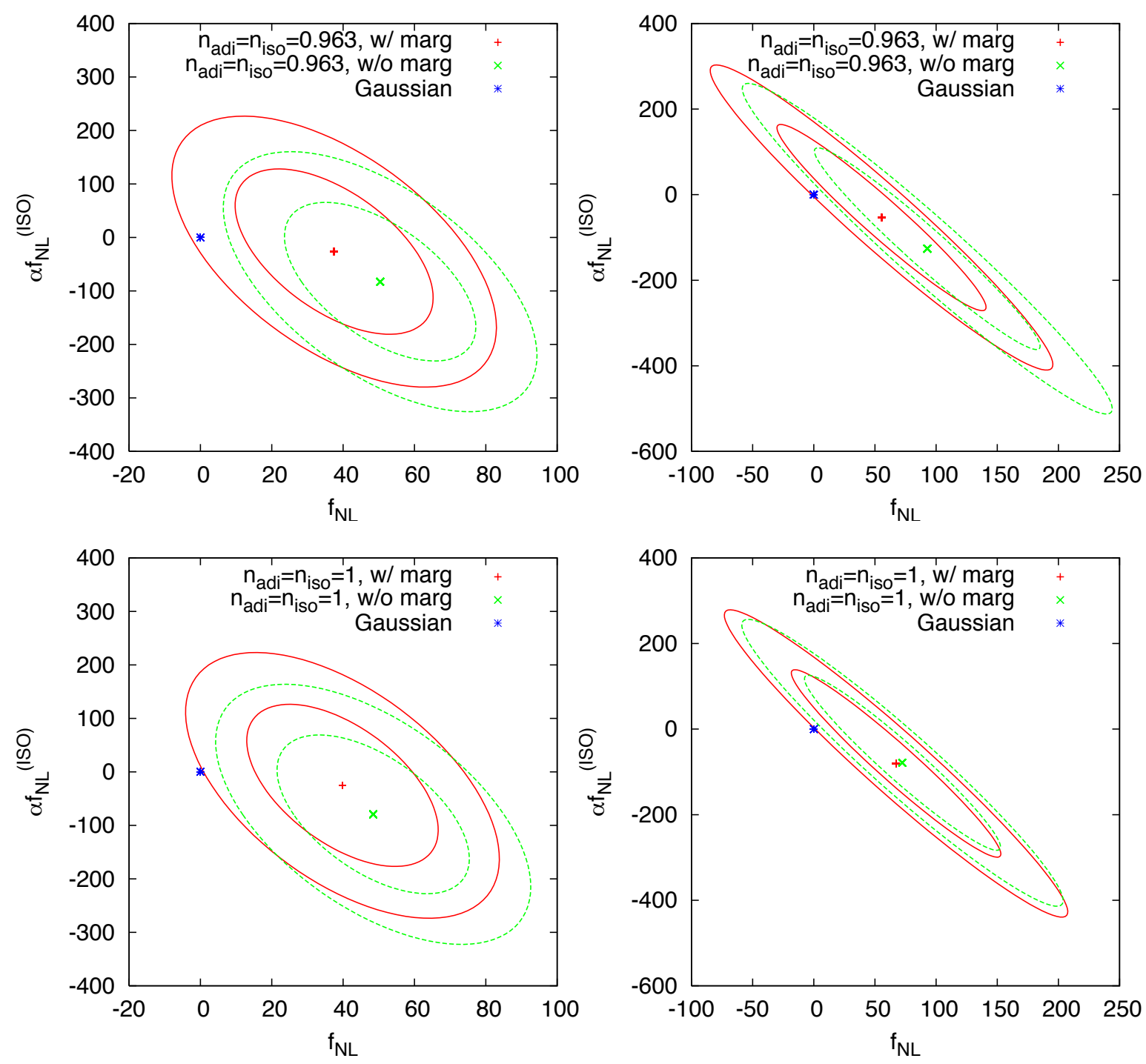

Figure 2: Joint constraints on $f_{\mathrm{NL}}$ and $\alpha f_{\mathrm{NL}}^{(\mathrm{ISO})}$ at 1 and $2 \sigma$ levels for correlated isocurvature models. Left and right panels show the constraints for the cases of CI and NID modes. We here assume $n_{\text {adi }}=n_{\text {iso }}$ and cases of $n_{\text {adi }}=n_{\text {iso }}=0.963$ and 1 are respectively shown in top and bottom panels. Other conventions are the same as in Fig. 1. 


\begin{tabular}{llcc}
\hline \hline & setups & $f_{\mathrm{NL}}$ & $\alpha f_{\mathrm{NL}}^{(\mathrm{ISO})}$ \\
\hline $\mathrm{CI}, n_{\text {iso }}=n_{\text {adi }}=0.963$ & $\mathrm{w} /$ o template marginalization & $41 \pm 21$ & $76 \pm 114$ \\
& & $(50 \pm 25)$ & $(-82 \pm 138)$ \\
\cline { 2 - 4 } & $\mathrm{w} /$ template marginalization & $34 \pm 21$ & $90 \pm 120$ \\
& $(37 \pm 25)$ & $(-26 \pm 144)$ \\
\hline $\mathrm{CI}, n_{\text {iso }}=n_{\text {adi }}=1$ & $\mathrm{w} /$ o template marginalization & $40 \pm 21$ & $70 \pm 114$ \\
& & $(48 \pm 25)$ & $(-79 \pm 138)$ \\
\cline { 2 - 4 } & $\mathrm{w} /$ template marginalization & $37 \pm 21$ & $99 \pm 117$ \\
& & $(40 \pm 25)$ & $(-25 \pm 141)$ \\
\hline NID, $n_{\text {iso }}=n_{\text {adi }}=0.963$ w/o template marginalization & $45 \pm 21$ & $103 \pm 55$ \\
& & $(93 \pm 86)$ & $(-126 \pm 220)$ \\
\cline { 2 - 4 } & $\mathrm{w} /$ template marginalization & $35 \pm 21$ & $82 \pm 54$ \\
$\mathrm{NID}, n_{\text {iso }}=n_{\text {adi }}=1$ & $\mathrm{w} /$ o template marginalization & $42 \pm 21$ & $99 \pm 53$ \\
& & $(72 \pm 75)$ & $(-78 \pm 191)$ \\
\cline { 2 - 4 } & $\mathrm{w} /$ template marginalization & $36 \pm 21$ & $86 \pm 53$ \\
& & $(67 \pm 80)$ & $(-80 \pm 204)$ \\
\hline \hline
\end{tabular}

Table 5: Constraints on $f_{\mathrm{NL}}$ and $\alpha f_{\mathrm{NL}}^{(\mathrm{ISO})}$ for the cases of correlated isocurvature perturbations.

\section{Implications to the curvaton scenario}

In this section, we demonstrate an application of our result to a model of the very early Universe based on the curvaton scenario. We here focus on a model where inflaton and curvaton fields source the primordial curvature and CDM isocurvature perturbations. In addition, we assume that some fraction of CDM is generated before curvaton decay, and the rest of CDM is generated directly from the curvaton decay. No CDM production after curvaton decay is assumed. Here we briefly outline the model and its predictions for the power spectrum and non-linearity parameters. For more details, we refer to e.g. Refs. [18, 20-22].

Adopting the sudden decay approximation, the curvaton decays synchronously on the uniform-density hypersurface of the total matter. On this hypersurface, the energy conservation of the total matter and CDM should be given by

$$
\begin{aligned}
\bar{\rho}_{r} & =\bar{\rho}_{r}^{(\phi)} e^{4\left(\zeta^{(\phi)}-\zeta\right)}+\bar{\rho}_{\sigma} e^{3\left(\zeta_{\sigma}-\zeta\right)} \\
\bar{\rho}_{\mathrm{CDM}} e^{3\left(\zeta_{\mathrm{CDM}}-\zeta\right)} & =\bar{\rho}_{\mathrm{CDM}}^{(\phi)} e^{3\left(\zeta^{(\phi)}-\zeta\right)}+\bar{\rho}_{\mathrm{CDM}}^{(\sigma)} e^{3\left(\zeta_{\sigma}-\zeta\right)}
\end{aligned}
$$

where $\rho_{r}^{(\phi)}$ and $\rho_{\sigma}$ are respectively energy densities of radiation and curvaton just before decay. The subscript $\phi$ for $\rho_{r}^{(\phi)}$ is put to emphasize that the radiation originates from the inflaton decay (or decay products). $\rho_{r}$ and $\rho_{\mathrm{CDM}}$ are respectively ones for radiation and 
CDM after the decay. $\rho_{\mathrm{CDM}}^{(\phi)}$ and $\rho_{\mathrm{CDM}}^{(\sigma)}$ are respectively energy densities of CDM produced from the inflaton and the curvaton, which leads to $\bar{\rho}_{\mathrm{CDM}}=\bar{\rho}_{\mathrm{CDM}}^{(\phi)}+\bar{\rho}_{\mathrm{CDM}}^{(\sigma)} \cdot \zeta^{(\phi)}$ is the curvature perturbation generated from the inflaton. $\zeta_{\sigma}$ and $\zeta_{\mathrm{CDM}}$ respectively are the ones on uniform curvaton and CDM energy density hypersurfaces. $\zeta$ is for the total one.

In the first equation, we assumed that CDM is energetically negligible during the decay and also the Universe is almost completely dominated by radiation after the curvaton decay. Otherwise, the Universe is dominated by matter soon after its decay, which would require further entropy production.

To describe the model, we introduce some parameters. We define the fractions of energy densities created from the curvaton decay in radiation and CDM as $\epsilon_{r}$ and $\epsilon_{\mathrm{CDM}}$, respectively, i.e.

$$
\epsilon_{r} \equiv \bar{\rho}_{\sigma} / \bar{\rho}_{r}, \quad \epsilon_{\mathrm{CDM}} \equiv \bar{\rho}_{\mathrm{CDM}}^{(\sigma)} / \bar{\rho}_{\mathrm{CDM}} .
$$

Then Eqs. (43) and (44) can be recast into

$$
\begin{aligned}
1 & =\left(1-\epsilon_{r}\right) e^{4\left(\zeta^{(\phi)}-\zeta\right)}+\epsilon_{r} e^{3\left(\zeta_{\sigma}-\zeta\right)} \\
e^{3\left(\zeta_{\mathrm{CDM}}-\zeta\right)} & =\left(1-\epsilon_{\mathrm{CDM}}\right) e^{3\left(\zeta^{(\phi)}-\zeta\right)}+\epsilon_{\mathrm{CDM}} e^{3\left(\zeta_{\sigma}-\zeta\right)}
\end{aligned}
$$

By solving Eqs. (46) and (47) for the curvature perturbation $\zeta$ and CDM isocurvature fluctuations $S_{\mathrm{CDM}}=3\left(\zeta_{\mathrm{CDM}}-\zeta\right)$ up to second order in $\zeta^{(\phi)}$ and $S_{\sigma}=3\left(\zeta_{\sigma}-\zeta^{(\phi)}\right)$, we obtain

$$
\begin{aligned}
\zeta= & \zeta^{(\phi)}+\frac{r S_{\sigma}}{3}+\frac{(1-r)(3+r)}{2 r}\left(\frac{r S_{\sigma}}{3}\right)^{2}, \\
S_{\mathrm{CDM}}= & {\left[\frac{\epsilon_{\mathrm{CDM}}}{r}-1\right] r S_{\sigma} } \\
& +\left[\frac{\epsilon_{\mathrm{CDM}}\left(1-\epsilon_{\mathrm{CDM}}\right)}{2 r^{2}}-\frac{(1-r)(3+r)}{6 r}\right]\left(r S_{\sigma}\right)^{2},
\end{aligned}
$$

where $r$ is defined as

$$
r \equiv \frac{3 \epsilon_{r}}{4-\epsilon_{r}}
$$

Furthermore, $S_{\sigma}$ can be expanded up to the second order as, assuming that the curvaton has a quadratic potential,

$$
S_{\sigma}=2 \frac{\delta \sigma_{*}}{\sigma_{*}}-\left(\frac{\delta \sigma_{*}}{\sigma_{*}}\right)^{2},
$$

where $\sigma_{*}=\bar{\sigma}_{*}+\delta \sigma_{*}$ is the value of the curvaton at the time of horizon exit and its fluctuations $\delta \sigma_{*}$ is assumed to be Gaussian. We also treat $\zeta^{(\phi)}$ as a Gaussian field.

Although our setup here a bit differs from one in Section 2, where curvature and isocurvature perturbations are assumed to be generated from a single source, our result in Section 4 for correlated isocurvature models can be still applicable as bispectra are 
generated from the single source $S_{\sigma}$. Then $\alpha$ and the parameters characterizing amplitudes of bispectra, $f_{\mathrm{NL}}$ and $\alpha f_{\mathrm{NL}}^{(\mathrm{ISO})}$, are given by

$$
\begin{aligned}
\alpha & =\frac{9}{A}\left[\frac{\epsilon_{\mathrm{CDM}}}{r}-1\right]^{2}, \\
f_{\mathrm{NL}} & =\frac{5(1-r)(3+r)}{6 r A^{2}}, \\
\alpha f_{\mathrm{NL}}^{(\mathrm{ISO})} & =\frac{9}{A^{2}}\left[\frac{\epsilon_{\mathrm{CDM}}\left(1-\epsilon_{\mathrm{CDM}}\right)}{2 r^{2}}-\frac{(1-r)(3+r)}{6 r}\right],
\end{aligned}
$$

where we defined a parameter $A$, which is the ratio of total curvature power to the contribution of the curvaton therein, i.e.

$$
A \equiv \frac{\left\langle\zeta^{2}\right\rangle}{\left\langle\left(r S_{\sigma} / 3\right)^{2}\right\rangle} .
$$

Fig. 3 shows constraints on $r$ and $\epsilon_{\mathrm{CDM}}$ at $2 \sigma$ level with several fixed values of $A$. Shaded regions are allowed by the constraints $\alpha, f_{\mathrm{NL}}$ and $\alpha f_{\mathrm{NL}}^{(\mathrm{ISO})}$. Regarding the upper bound on $\alpha$, when $A \gg 1, S_{\mathrm{CDM}}$ is almost uncorrelated with the curvature perturbation since it almost originates to that from the inflaton. Thus Eq. (13) is adopted. As for bounds on $f_{\mathrm{NL}}$ and $f_{\mathrm{NL}}^{(\mathrm{ISO})}$, we adopt those for correlated models with $n_{\text {adi }}=n_{\text {iso }}=1$ and template marginalization, $f_{\mathrm{NL}}<90$ and $-307<\alpha f_{\mathrm{NL}}^{(\mathrm{ISO})}<257$.

When $A$ is as large as $\mathcal{O}(100)$, there is some parameter region where $\alpha f_{\mathrm{NL}}^{(\mathrm{ISO})}$ can be observably large as $\alpha f_{\mathrm{NL}}^{(\mathrm{ISO})}=\mathcal{O}(100)$ without producing too large $\alpha$ and $f_{\mathrm{NL}}$. In such a case, the constraints on $\alpha f_{\mathrm{NL}}^{(\mathrm{ISO})}$ becomes important.

\section{Conclusion}

In this paper, we have investigated non-Gaussianties in isocurvature fluctuations by extending our previous analysis for CDM uncorrelated mode [25] to a more general type. We derived optimal constraints on them using the CMB temperature maps of the WMAP 7-year observation. Specifically, we have studied correlated/uncorrelated CDM and neutrino density isocurvature modes along with the counterpart for the adiabatic one. Their constraints are summarized in Tables 4 and 5 for uncorrelated and correlated modes, respectively. We have also presented joint analysis in which both the adiabatic and isocurvature fluctuations are assumed to exist and obtained 2D constraints as shown in Figs. 1 and 2 for uncorrelated and correlated cases, respectively. We have checked our results by making the Fisher matrix analysis adopting the specifications of WMAP7 experiments, which should give an idea how large the errors can be. Comparing with the results obtained by using actual CMB temperature maps, we have found that they are almost the same in size, which shows the reasonability of our constraints.

Our results indicate that both of the adiabatic and isocurvature perturbations, even if we consider various types for the latter, are consistent with Gaussian ones at around $2 \sigma$ 

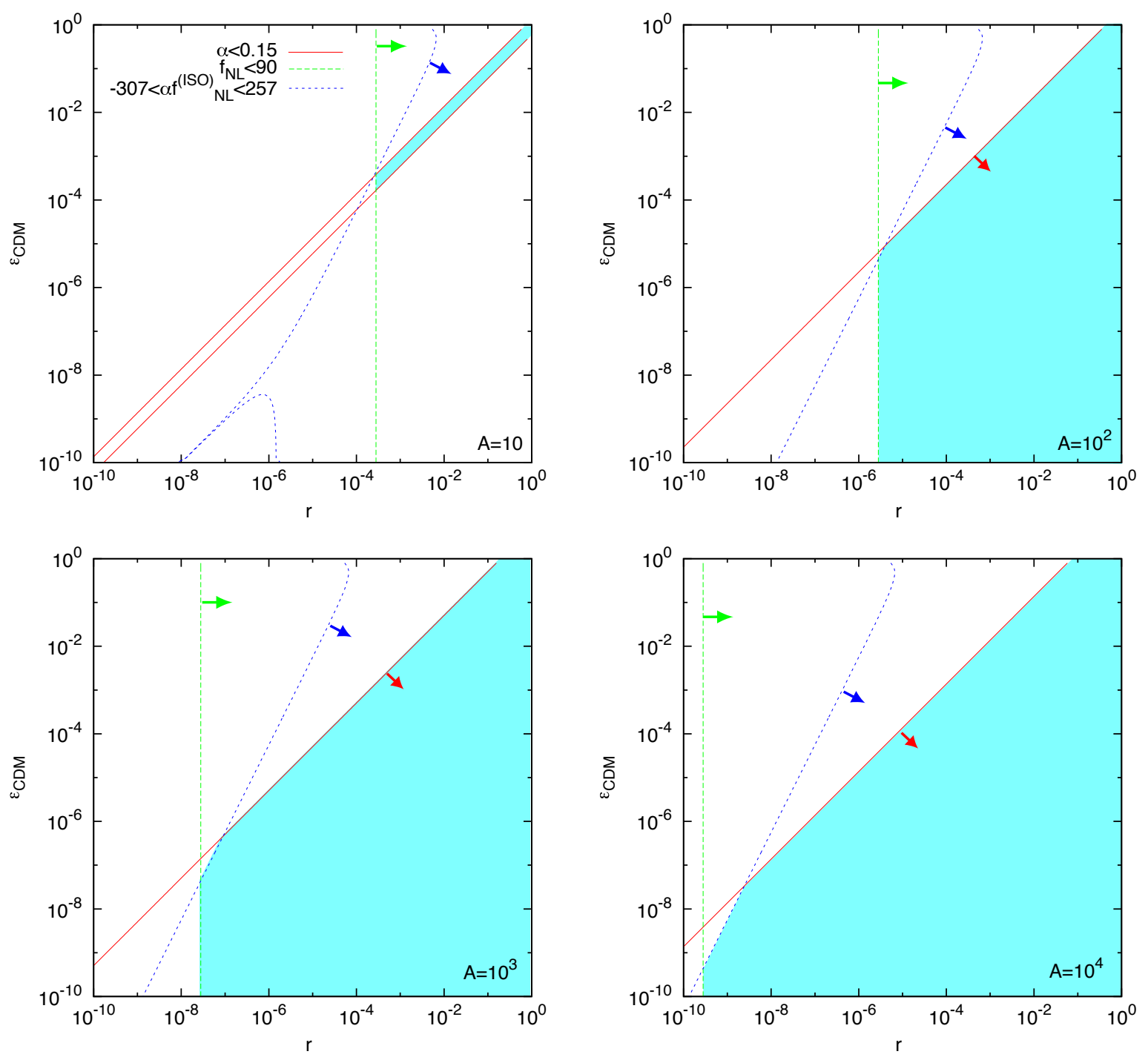

Figure 3: Constraints on curvaton scenario in $r-\epsilon_{\mathrm{CDM}}$ plane with fixed values of $A$. From top left to bottom right panels, $A$ scales from 10 to $10^{4}$. An arrow indicates the direction of which region is allowed for each constraints. Shaded regions are allowed by the constraints $\alpha, f_{\mathrm{NL}}$ and $\alpha f_{\mathrm{NL}}^{(\mathrm{ISO})}$. For the case of $A=10$, the region allowed by the constraints from $\alpha$ is the one between two red lines. See text for more details. 
level. Although we have not detected any non-Gaussian signatures in those perturbations, their constraints may give interesting implications to some explicit models. As such an example, we have considered a curvaton scenario where correlated CDM fluctuations are generated. As mentioned in the text, isocurvature fluctuations have already been severely constrained by power spectrum measurements. However, we argued that, in some particular cases, our constraints for isocurvature non-Gaussianities can give a more stringent one.

In the near future, we are expecting to obtain much more accurate measurements of CMB from Planck. At this era of precision cosmology, isocurvature fluctuations and their non-Gaussianities would provide fruitful information on the physics of the early Universe and the research along this line may also give important implications to particle physics as well.

\section{Acknowledgment}

T. S. would like to thank the Japan Society for the Promotion of Science for the financial report. The authors acknowledge Kobayashi-Maskawa Institute for the Origin of Particles and the Universe, Nagoya University for providing computing resources in conducting the research reported in this paper. This work is supported by Grant-in-Aid for Scientific research from the Ministry of Education, Science, Sports, and Culture (MEXT), Japan, No. 14102004 (M.K.), No. 21111006 (M.K.), No. 23740195 (T.T.), No. 24740160 (C.H.) and also by World Premier International Research Center Initiative (WPI Initiative), MEXT,

Japan. Some of the results in this paper have been derived using the HEALPix [75] package.

\section{References}

[1] R. Bean, J. Dunkley and E. Pierpaoli, Phys. Rev. D 74, 063503 (2006) [astro$\mathrm{ph} / 0606685]$.

[2] K. Moodley, M. Bucher, J. Dunkley, P. G. Ferreira and C. Skordis, Phys. Rev. D 70, 103520 (2004) [astro-ph/0407304].

[3] M. Beltran, J. Garcia-Bellido, J. Lesgourgues and A. Riazuelo, Phys. Rev. D 70, 103530 (2004) [astro-ph/0409326].

[4] R. Trotta, Mon. Not. Roy. Astron. Soc. 375, L26 (2007) [astro-ph/0608116].

[5] R. Keskitalo, H. Kurki-Suonio, V. Muhonen and J. Valiviita, JCAP 0709, 008 (2007) [astro-ph/0611917]. 
[6] M. Kawasaki and T. Sekiguchi, Prog. Theor. Phys. 120, 995 (2008) [arXiv:0705.2853 [astro-ph]].

[7] I. Sollom, A. Challinor and M. P. Hobson, Phys. Rev. D 79, 123521 (2009) [arXiv:0903.5257 [astro-ph.CO]].

[8] J. Valiviita and T. Giannantonio, Phys. Rev. D 80, 123516 (2009) [arXiv:0909.5190 [astro-ph.CO]].

[9] H. Li, J. Liu, J. -Q. Xia and Y. -F. Cai, Phys. Rev. D 83, 123517 (2011) [arXiv:1012.2511 [astro-ph.CO]].

[10] J. Valiviita, M. Savelainen, M. Talvitie, H. Kurki-Suonio and S. Rusak, Astrophys. J. 753, 151 (2012) [arXiv:1202.2852 [astro-ph.CO]].

[11] C. Gordon and J. R. Pritchard, Phys. Rev. D 80, 063535 (2009) [arXiv:0907.5400 [astro-ph.CO]].

[12] M. Kawasaki, T. Sekiguchi and T. Takahashi, JCAP 1110, 028 (2011) [arXiv:1104.5591 [astro-ph.CO]].

[13] C. Hikage, D. Munshi, A. Heavens and P. Coles, Mon. Not. Roy. Astron. Soc. 404, 1505 (2010) [arXiv:0907.0261 [astro-ph.CO]].

[14] D. Langlois and B. van Tent, Class. Quant. Grav. 28, 222001 (2011) [arXiv:1104.2567 [astro-ph.CO]].

[15] D. Langlois and B. van Tent, JCAP 1207, 040 (2012) [arXiv:1204.5042 [astro-ph.CO]].

[16] E. Kawakami, M. Kawasaki, K. Miyamoto, K. Nakayama and T. Sekiguchi, JCAP 1207, 037 (2012) [arXiv:1202.4890 [astro-ph.CO]].

[17] E. Kawakami, M. Kawasaki, K. Nakayama and F. Takahashi, JCAP 0909, 002 (2009) [arXiv:0905.1552 [astro-ph.CO]].

[18] D. Langlois, F. Vernizzi and D. Wands, JCAP 0812, 004 (2008) [arXiv:0809.4646 [astro-ph]].

[19] T. Takahashi, M. Yamaguchi and S. Yokoyama, Phys. Rev. D 80, 063524 (2009) [arXiv:0907.3052 [astro-ph.CO]].

[20] D. Langlois and A. Lepidi, JCAP 1101, 008 (2011) [arXiv:1007.5498 [astro-ph.CO]].

[21] D. Langlois and T. Takahashi, JCAP 1102, 020 (2011) [arXiv:1012.4885 [astroph.CO]].

[22] M. Kawasaki, K. Nakayama, T. Sekiguchi, T. Suyama and F. Takahashi, JCAP 0901, 042 (2009) [arXiv:0810.0208 [astro-ph]]. 
[23] M. Kawasaki, K. Nakayama, T. Sekiguchi, T. Suyama and F. Takahashi, JCAP 0811, 019 (2008) [arXiv:0808.0009 [astro-ph]].

[24] C. Hikage, K. Koyama, T. Matsubara, T. Takahashi and M. Yamaguchi, Mon. Not. Roy. Astron. Soc. 398, 2188 (2009) [arXiv:0812.3500 [astro-ph]].

[25] C. Hikage, M. Kawasaki, T. Sekiguchi and T. Takahashi, arXiv:1211.1095 [astroph.CO].

[26] M. Axenides, R. H. Brandenberger, M. S. Turner, Phys. Lett. B126, 178 (1983).

[27] D. Seckel, M. S. Turner, Phys. Rev. D32, 3178 (1985).

[28] A. D. Linde, Phys. Lett. B158, 375-380 (1985).

[29] A. D. Linde, D. H. Lyth, Phys. Lett. B246, 353-358 (1990).

[30] M. S. Turner, F. Wilczek, Phys. Rev. Lett. 66, 5 (1991).

[31] A. D. Linde, Phys. Lett. B259, 38 (1991).

[32] D. H. Lyth, Phys. Rev. D45, 3394 (1992).

[33] S. Kasuya, M. Kawasaki, Phys. Rev. D80, 023516 (2009) [arXiv:0904.3800 [astroph.CO]].

[34] K. Enqvist and J. McDonald, Phys. Rev. Lett. 83, 2510 (1999) [arXiv:hep$\mathrm{ph} / 9811412]$.

[35] K. Enqvist, J. McDonald, Phys. Rev. D62, 043502 (2000) [hep-ph/9912478].

[36] M. Kawasaki and F. Takahashi, Phys. Lett. B 516, 388 (2001) [arXiv:hep$\mathrm{ph} / 0105134]$.

[37] K. Enqvist and M. S. Sloth, Nucl. Phys. B 626, 395 (2002) [arXiv:hep-ph/0109214].

[38] D. H. Lyth and D. Wands, Phys. Lett. B 524, 5 (2002) [arXiv:hep-ph/0110002].

[39] T. Moroi and T. Takahashi, Phys. Lett. B 522, 215 (2001) [Erratum-ibid. B 539, 303 (2002)] [arXiv:hep-ph/0110096].

[40] T. Moroi and T. Takahashi, Phys. Lett. B 671, 339 (2009) [arXiv:0810.0189 [hep-ph]].

[41] T. Moroi and T. Takahashi, Phys. Rev. D 66, 063501 (2002) [arXiv:hep-ph/0206026].

[42] T. Moroi and H. Murayama, Phys. Lett. B 553, 126 (2003) [hep-ph/0211019].

[43] D. H. Lyth, C. Ungarelli and D. Wands, Phys. Rev. D 67, 023503 (2003) [arXiv:astro$\mathrm{ph} / 0208055]$. 
[44] D. H. Lyth and D. Wands, Phys. Rev. D 68, 103516 (2003) [arXiv:astro-ph/0306500].

[45] K. Hamaguchi, M. Kawasaki, T. Moroi and F. Takahashi, Phys. Rev. D 69, 063504 (2004) [hep-ph/0308174].

[46] C. Gordon and K. A. Malik, Phys. Rev. D 69, 063508 (2004) [astro-ph/0311102].

[47] M. Ikegami and T. Moroi, Phys. Rev. D 70, 083515 (2004) [hep-ph/0404253].

[48] F. Ferrer, S. Rasanen and J. Valiviita, JCAP 0410, 010 (2004) [astro-ph/0407300].

[49] M. Beltran, Phys. Rev. D 78, 023530 (2008) [arXiv:0804.1097 [astro-ph]].

[50] M. Lemoine, J. Martin and J. 'i. Yokoyama, Europhys. Lett. 89, 29001 (2010) [arXiv:0903.5428 [astro-ph.CO]].

[51] M. Lemoine, J. Martin and J. 'i. Yokoyama, Phys. Rev. D 80, 123514 (2009) [arXiv:0904.0126 [astro-ph.CO]].

[52] M. Bucher, K. Moodley and N. Turok, Phys. Rev. D 62, 083508 (2000) [astro$\mathrm{ph} / 9904231]$.

[53] M. Sasaki and E. D. Stewart, Prog. Theor. Phys. 95, 71 (1996) [astro-ph/9507001].

[54] D. H. Lyth, K. A. Malik and M. Sasaki, JCAP 0505, 004 (2005) [astro-ph/0411220].

[55] D. H. Lyth and Y. Rodriguez, Phys. Rev. D 71, 123508 (2005) [astro-ph/0502578].

[56] D. H. Lyth and Y. Rodriguez, Phys. Rev. Lett. 95, 121302 (2005) [astro-ph/0504045].

[57] M. Kawasaki, K. Nakayama and F. Takahashi, JCAP 0901, 002 (2009) [arXiv:0809.2242 [hep-ph]].

[58] E. Komatsu et al. [WMAP Collaboration], Astrophys. J. Suppl. 192, 18 (2011) [arXiv:1001.4538 [astro-ph.CO]].

[59] J. Dunkley, R. Hlozek, J. Sievers, V. Acquaviva, P. A. R. Ade, P. Aguirre, M. Amiri and J. W. Appel et al., Astrophys. J. 739, 52 (2011) [arXiv:1009.0866 [astro-ph.CO]].

[60] M. Kawasaki, K. Miyamoto, K. Nakayama and T. Sekiguchi, JCAP 1202, 022 (2012) [arXiv:1107.4962 [astro-ph.CO]].

[61] E. Komatsu and D. N. Spergel, Phys. Rev. D 63, 063002 (2001) [astro-ph/0005036].

[62] D. Babich and M. Zaldarriaga, Phys. Rev. D 70, 083005 (2004) [astro-ph/0408455].

[63] A. Lewis, A. Challinor and A. Lasenby, Astrophys. J. 538, 473 (2000) [astro$\mathrm{ph} / 9911177]$. 
[64] L. Knox, Phys. Rev. D 52, 4307 (1995) [astro-ph/9504054].

[65] M. Limon, "Wilkinson Microwave Anisotropy Probe (WMAP): Seven Year Explanatory Supplement," http://lambda.gsfc.nasa.gov/product/map/current/

[66] E. Komatsu, D. N. Spergel and B. D. Wandelt, Astrophys. J. 634, 14 (2005) [astro$\mathrm{ph} / 0305189]$.

[67] A. P. S. Yadav, E. Komatsu and B. D. Wandelt, Astrophys. J. 664, 680 (2007) [astro-ph/0701921].

[68] P. Creminelli, A. Nicolis, L. Senatore, M. Tegmark and M. Zaldarriaga, JCAP 0605, 004 (2006) [astro-ph/0509029].

[69] A. P. S. Yadav, E. Komatsu, B. D. Wandelt, M. Liguori, F. K. Hansen and S. Matarrese, Astrophys. J. 678, 578 (2008) [arXiv:0711.4933 [astro-ph]].

[70] K. M. Smith, L. Senatore and M. Zaldarriaga, JCAP 0909, 006 (2009) [arXiv:0901.2572 [astro-ph]].

[71] F. Elsner and B. D. Wandelt, Astrophys. J. Suppl. 184, 264 (2009) [arXiv:0909.0009 [astro-ph.CO]].

[72] K. M. Smith, O. Zahn and O. Dore, Phys. Rev. D 76, 043510 (2007) [arXiv:0705.3980 [astro-ph]].

[73] N. Jarosik, C. L. Bennett, J. Dunkley, B. Gold, M. R. Greason, M. Halpern, R. S. Hill and G. Hinshaw et al., Astrophys. J. Suppl. 192, 14 (2011) [arXiv:1001.4744 [astroph.CO]].

[74] B. Gold, N. Odegard, J. L. Weiland, R. S. Hill, A. Kogut, C. L. Bennett, G. Hinshaw and J. Dunkley et al., Astrophys. J. Suppl. 192, 15 (2011) [arXiv:1001.4555 [astroph.GA]].

[75] K. M. Gorski, E. Hivon, A. J. Banday, B. D. Wandelt, F. K. Hansen, M. Reinecke and M. Bartelman, Astrophys. J. 622, 759 (2005) [astro-ph/0409513]. 